\section{A molecular handle on the Neanderthals}

Ryk Ward and Chris Stringer

The sequencing of mitochondrial DNA from Neanderthal fossil bone is a terrific achievement. Among the conclusions to be drawn from the data is that there was a period of more than 500,000 years during which Neanderthals and the line leading to modern humans evolved independently.

$\mathrm{N}$ eanderthals hold a central place in studies of human evolution, yet their relationship to contemporary humans remains enigmatic ${ }^{1,2}$. Now, a molecular analysis has seemingly resolved many of the disputes about that relationship. The work, reported in last week's Cell ${ }^{3}$, represents the first successful recovery and analysis of mitochondrial DNA (mtDNA) from a Neanderthal specimen. Even more remarkably, the sequence was recovered from the first Neanderthal skeleton described, the type specimen, one of the most important human fossils ever found.

The type-specimen Neanderthal skeleton was discovered in the Neander Valley, near Dusseldorf in Germany, and described in 1857 (see refs 1 and 2 for an overview). This event ignited an intense debate about the degree of morphological distinctiveness - and biological continuity - between ancient and modern humans. Some early workers believed that the peculiar aspects of Neanderthal morphology were due to skeletal diseases. But additional finds made it clear that such remains represented a population of archaic humans which inhabited Europe and western Asia between about 230,000 and 30,000 years ago.

In agreement with what we know of their environments and lifestyle, their physique was muscular and thickset, with body proportions apparently reflecting adaptation to cold conditions and with a skeleton built for strength and endurance. Although the morphological diversity of the Neanderthal population was probably at least as great as that of modern humans, overall they were characterized by a number of distinctive anatomical features ${ }^{1,2}$.

More recently, even older types of archaic humans have been found that are morphologically distinct from both Neanderthals and contemporary human populations. Their presence in Europe and Africa only serves to increase the complexity of untangling the threads of human evolution. As far as Neanderthals are concerned, it is difficult to find consistent morphological antecedents in these early remains. Although some workers claim that Neanderthal morphology runs back to the early Middle Pleistocene, some 600,000-800,000 years ago, others argue for their genetic continuity with modern humans. An intermediate position is that a Mid-Pleistocene species (Homo heidelbergensis), which occurred in Africa and Europe, gave rise to both Neanderthals and modern humans $s^{4}$.

The new molecular work ${ }^{3}$ considerably clarifies matters. But it is first necessary to realize that the difficulty of working with ancient DNA (see box, overleaf) required many procedures to generate the primary data. The first step evaluated the amount of amino-acid racemization, as a surrogate measure of the amount of damage likely to have been suffered by the Neanderthal DNA. Although the degree of racemization of aspartic acid was approximately double that of modern bone (indicating some degradation), the results lay within the range of feasible amplification with the polymerase chain reaction. Two independent PCR reactions were accordingly performed, using primers designed to amplify a short, 105base-pair segment of the hypervariable segment of the human mtDNA control region. In each case, a PCR product was obtained and these were cloned into plasmid vectors and 30 clones sequenced.

The resulting sequences indicated that the PCR products contained a mixture of molecules (see box): a small minority population of modern human sequences, presumably contaminants, and a greater number of highly distinctive sequences, tentatively identified as the Neanderthal sequence. The putative Neanderthal sequences were characterized by the presence of eight deviations from the reference sequence (out of 61 positions). As the same set of eight substitutions was obtained on two further PCR amplifications, it was highly likely that this sequence came from the Neanderthal fossil.

Because primers specifically designed to amplify the putative Neanderthal sequence (rather than modern human mtDNA) failed to yield products from a sample of modern humans, the possibility that the variant sequence might be a nuclear insert could be eliminated. Finally, and perhaps most importantly, the putative Neanderthal sequence was independently replicated in a collaborating laboratory, so this mtDNA

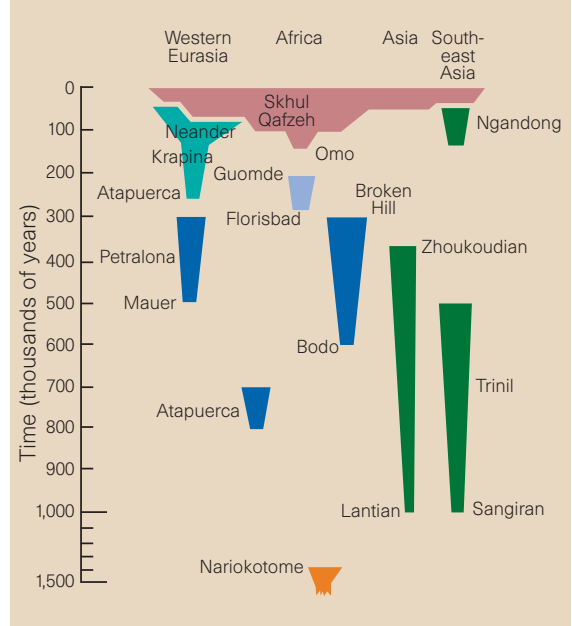

Figure 1 Distribution in time and space of the various human lineages that evolved from Homo ergaster (orange), the first expansion leading to the establishment and subsequent development of $H$. erectus lineages (green) in Asia and Southeast Asia. Other lineages include three groups of $H$. heidelbergensis (dark blue), Neanderthals (turquoise), a transitional group of archaic H. sapiens in Africa (light blue), and modern $H$. sapiens (mauve), the latter ultimately eclipsing all other human lineages. The new Neanderthal mtDNA results ${ }^{3}$ support the hypothesis that the original divergence that eventually led to Neanderthals and modern humans began more than 500,000 years ago in $H$. heidelbergensis. Places mentioned are sites of significant fossil discoveries.

sequence is almost certainly derived from the Neanderthal specimen itself.

To characterize a longer sequence, one likely to be more informative in evolutionary terms, a series of 13 independent overlapping amplifications (see box) was carried out, and the products cloned. After aligning the resulting set of 123 sequences from individual clones, a consensus sequence of 379 base pairs was obtained. This sequence exhibited 27 differences from the reference sequence, compared to the 5-8 differences expected from a random sample of modern humans. Detailed comparisons of the Neanderthal sequence with 994 human mtDNA lineages and 16 chimpanzee mtDNA lineages indicated that, although human mtDNA lineages differed among themselves by $8.0 \pm 3.1$ substitutions, the difference between humans and the Neanderthal sequence was $27 \pm 2.2$. The overall difference between the Neanderthal sequence and that of humans was about three times the average difference among humans, but only about half the difference between humans and chimpanzees.

This implies that the Neanderthal divergence is of considerable antiquity, dating to 555,000 to 690,000 years ago. This is about four times greater than the time back to the common ancestor of modern human mtDNA (120,000 to 150,000 years). 


\section{Standards for research on ancient DNA}

It is the development of sound experimental procedures, rather than the delivery of breathtaking but dubious results, that has represented the key to establishing the credibility of research on ancient DNA. Aside from taking due care with the extraction procedure, and controlling for contamination, four essential experimental procedures should be carried out.

- Determine the amount of change that has occurred and resulted in damaged or degraded DNA. Because the rate at which L-amino acids undergo racemization to Damino acids is dependent on much the same factors that induce DNA damage (water, temperature), the $D / L$ ratio of amino acids can be used as an indirect measure of the extent of DNA damage?. Typically, a D/L ratio greater than 0.08 suggests that DNA cannot be reliably retrieved from the sample.

- Determine the number of intact template molecules in the polymerase chain reaction. Too few template
Position:

Reference sequence:

$123 \quad 3 \quad 4 \quad 5 \quad 6 \quad 7 \quad 899101112131415$

\section{Clone}

A.1 . . C G . . G

A.2 G . C G . . $\mathbf{G}$

A.3 . . C G . . G

A.4 . C G . G G

A.5 . . . . .

B.1 C. G, C . T .

B.2 . G . C T T

B.3

B.4

B.5

C.1

C.2

C. 3

C. 4

C.5

Ancient DNA sequence

molecules will yield

unreliable and inconsistent results, because of the misincorporation of nucleotides and other artefacts of PCR. However, once the correct target has been identified, a diagnostic template can be synthesized and serial dilutions set up for quantitative PCR experiments, yielding estimates of the number of original template molecules ${ }^{8}$. $\mathrm{PCR}$ reactions with substantially fewer than
. $\mathrm{G} . \mathrm{C} \cdot \mathrm{T}$.

. G. C . T.

. . G C . .

$\mathrm{T} . \mathrm{T}$.

T. T A

$T \cdot T$.

$\mathrm{T} \cdot \mathrm{T}$.

. C. .

A C C G T T G A C C T T A T G 50-100 template molecules tend to be highly suspect.

- Use cloning to identify the composition of the PCR products, and to obtain a consensus sequence for the target. Cloning the PCR product $^{3}$ reveals its underlying nature and allows reconstruction of the ancient DNA sequence. The figure here illustrates five clones from each of three independent, overlapping, amplifications ( $A, B, C)$. In each amplification there is a minority sequence (A.5, B.5, C.5) resembling the reference, and a presumed contaminant. Alignment of the remaining 12 sequences allows reconstruction of the ancient DNA sequence and indicates that it deviates from the reference at six positions $(3,4,7,9,11,14)$. Note that the modern contaminant has a polymorphism at position 9 , and that there are six instances of nucleotide misincorporation (singleton bases in green).

- Use independent replication to confirm results. This is a standard experimental procedure, but all too often it has been ignored in work with ancient DNA.

The collective application of these four criteria not only marks the coming-of-age of research on ancient DNA, but serves as a useful yardstick for investigator and reviewer alike. In this light, the new results on Neanderthal mtDNA are merely a tantalizing indication of the landmark contributions that can be expected over the next decade. R.w.\& c.s.
More extensive analyses confirmed that the Neanderthal sequence consistently fell outside the mtDNA sequence variation observed in modern humans. They also suggested that the closest contemporary lineages to the Neanderthal sequence came from Africa. So the genetic relationship between Neanderthals and modern Europeans appears to be no closer than the average relationship between Neanderthals and any modern human - running counter to the view that Neanderthals were at least partly ancestral to modern Europeans ${ }^{5}$.

These molecular results, then, imply that there was a period of roughly half a million years of independent evolution of Neanderthals and the line leading to modern humans. This, in turn, suggests that the divergence might have occurred at about the time that the two main lines of Homo heidelbergensis became distinct in Europe and Africa respectively (Fig. 1). Such a scheme of events implies that the Mid-Pleistocene forms of $H$. heidelbergensis in Europe may have eventually led to early Neanderthals, such as the specimens from Atapuerca in Spain, and ultimately to the 'classic' forms found at Krapina, in Croatia, and at Neander itself. Conversely, modern humans are likely to have evolved from transitional groups of archaic Homo sapiens, such as those found at Guomde and Florisbad, in East and South Africa respectively, which in turn were independently derived from later $H$. heidelbergensis forms in Africa. Intriguingly, still other forms of humans may have survived in Southeast Asia ${ }^{6}$ (see Fig. 1).

With respect to the contentious issue of whether Neanderthals and anatomically early modern humans exchanged genes, these new results diminish, but do not rule out, that possibility. Clearly, Neanderthal populations represented by the type specimen did not contribute mtDNA to the modern human population. But, as the authors are careful to point out, this does not exclude the possibility of exchange of nuclear genes. In our view, whether we choose to designate them as separate species or not, half a million years of independent evolution of two highly specialized forms of human, with all the attendant behavioural implications, make such an exchange unlikely. Unfortunately, given the extremely low probability of obtaining nuclear DNA from the Neanderthal specimen, this is a hypothesis that may never be directly tested.

Given the quality of the new molecular findings, the study of ancient human DNA can, at long last, be said to be on a secure footing. As additional molecular data of increasing complexity are produced, the near future looks set to be a truly revolutionary time for students of human evolution.

Ryk Ward is at the Institute of Biological Anthropology, University of Oxford, Oxford OX2 $6 Q S, U K$. Chris Stringer is in the Department of Palaeontology, The Natural History Museum, London SW7 5BD, UK.

1. Stringer, C. \& Gamble, C. In Search of the Neanderthals (Thames \& Hudson, London, 1993).

2. Trinkaus, E. \& Shipman, P. The Neandertals (Cape, London, 1993).

3. Krings, M. et al. Cell 90, 19-30 (1997).

4. Gibbons, A. Science 27, 1331-1333 (1997).

5. Wolpoff, M. \& Caspari, R. Race and Human Evolution: A Fatal Attraction (Simon \& Schuster, New York, 1997).

6. Swisher, C. Science 274, 1870-1874 (1996).

7. Poinar, H. N., Höss, M., Bada, J. L. \& Pääbo, S. Science 272, 864-866 (1996).

8. Handt, O., Krings, M., Ward, R. H. \& Pääbo, S. Am. J. Hum. Genet. 59, 368-376 (1996). 